\title{
Research advances in chimeric antigen receptor- modified T-cell therapy (Review)
}

\author{
YUXI LUO ${ }^{1,2}$, GUIQIN SONG ${ }^{3}$, SHICHU LIANG $^{2}$, FEIFEI LI ${ }^{4}$ and KANG LIU ${ }^{1}$ \\ ${ }^{1}$ Institute of Tissue Engineering and Stem Cells, Nanchong Central Hospital, The Second Clinical Medical \\ College, North Sichuan Medical College, Nanchong, Sichuan 637000; ${ }^{2}$ The First Clinic of Anhui Medical \\ University, Hefei, Anhui 230032; ${ }^{3}$ Department of Biology, North Sichuan Medical College, Nanchong, Sichuan \\ 637000; ${ }^{4}$ Department of Pathophysiology, Anhui Medical University, Hefei, Anhui 230032, P.R. China
}

Received August 11, 2020; Accepted January 26, 2021

DOI: $10.3892 /$ etm.2021.9915

\begin{abstract}
Chimeric antigen receptor (CAR)-modified T-cells are T-cells that have been genetically engineered to express CAR molecules to target specific surface antigens on tumor cells. CAR T-cell therapy, a novel cancer immunotherapy, has been attracting increasing attention, since it exhibited notable efficacy in the treatment of hematological tumors in clinical trials. However, for this type of therapy, challenges must be overcome in the treatment of solid tumors. Furthermore, certain side effects associated with CAR T-cell therapy, including cytokine release syndrome, immune effector cellrelated neurotoxicity syndrome, tumor lysis syndrome and on-target off-tumor toxicity, must be taken into consideration. The present study provides a systematic review of the principle, clinical application, current challenges, possible solutions and future perspectives for CAR T-cell therapy.
\end{abstract}

\section{Contents}

1. Introduction

2. CAR T-cell therapy and the construction of CAR molecules

3. Clinical applications of CAR T-cell therapy

4 Current challenges and treatment principles of CAR T-cell therapy

5. Conclusions and outlook

Correspondence to: Professor Feifei Li, Department of Pathophysiology, Anhui Medical University, 81 Meishan Road, Hefei, Anhui 230032, P.R. China

E-mail: feifeili717@ahmu.edu.cn

Professor Kang Liu, Institute of Tissue Engineering and Stem Cells, Nanchong Central Hospital, The Second Clinical Medical College, North Sichuan Medical College, Renkang Building, 97 Renminnan Road, Nanchong, Sichuan 637000, P.R. China

E-mail: liukang@nsmc.edu.cn

Key words: chimeric antigen receptor-modified T-cells, immunotherapy, tumor, adoptive cellular therapy

\section{Introduction}

With the marked progress of molecular and cellular research and technology over the last few decades, notable advances have been made in the elucidation of the mechanisms underlying tumor development. The focus of cancer therapy has shifted from traditional therapy, including surgery, radiotherapy and chemotherapy (1), to biotherapy, including cancer immunotherapy, which was declared the annual scientific breakthrough in 2013 by Science (2).

Cancer immunotherapy has incomparable advantages over traditional cancer treatment, which may prolong the progression-free survival and overall survival of patients by unleashing an immune system attack against tumor cells in multiple targets and directions (3).

Immunotherapy, including immune checkpoint inhibitors, adoptive cellular therapy and tumor vaccines, is considered a milestone in cancer therapeutic strategies and is leading to a paradigm transformation in cancer management (4). Chimeric antigen receptor (CAR) T-cell therapy is a novel approach to cell adoptive immunotherapy after the advent of cell therapies, including the use of tumor-infiltrating lymphocytes, cytokine-induced killer (CIK) cells, natural killer cells, dendritic cells (DC)-CIK cells and T-cell receptorengineered T-cells.

To date, the US Food and Drug Administration (FDA) and European Medicines Agency have approved two CAR T-cell therapies, Novartis' Kymriah (tisagenlecleucel; CTL019) and Kite's Yescarta (Axicabtagene ciloleucel; KTE-C19) (5), of which Kymriah was aimed to treat relapsed B-cell acute lymphoblastic leukemia and Yescarta was indicated for the treatment of adult patients with relapsed or refractory large B-cell lymphoma (6). Furthermore, hundreds of novel CAR T-cell therapy clinical trials are underway worldwide with government support, indicating that CAR T-cell therapy has become widely accepted by both official agencies and the public due to its marked efficacy in treating certain malignancies $(7,8)$.

The aim of the present review was to provide an overview of the principle and clinical application of CAR T-cell therapies prior to addressing major challenges and proposing possible solutions and a future outlook. 


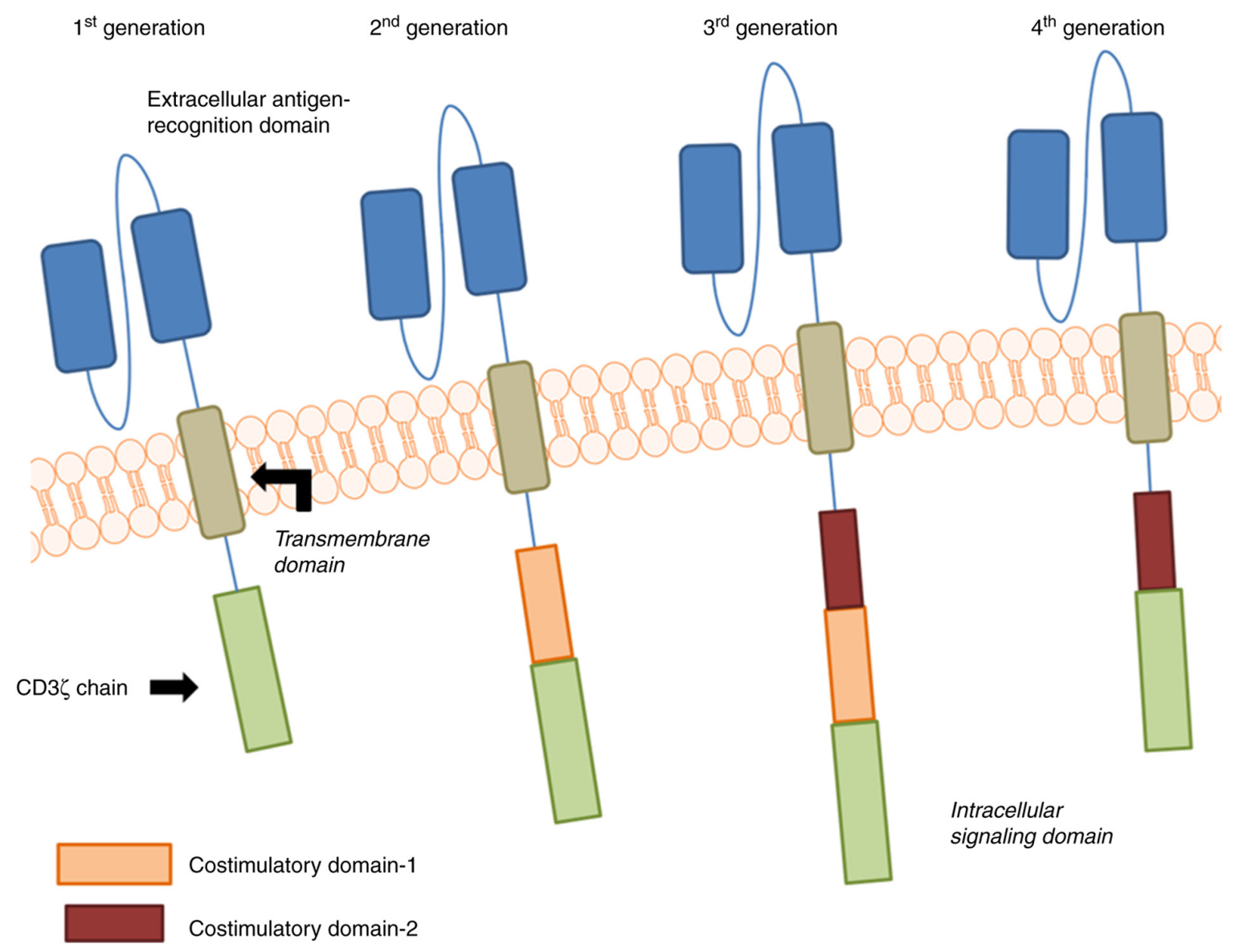

Figure 1. Schematic depiction of the structure of chimeric antigen receptor-modified molecules.

\section{CAR T-cell therapy and the construction of CAR molecules}

CAR T-cells are extracted autologous or allogeneic T-cells that have been genetically modified to express major histocompatibility complex-unrestricted and antigen-specific receptors on the surface, which are able to recognize and attack antigenbearing tumor cells after reinfusion (9). In brief, CAR T-cell therapy is a method that activates the patient's own immune system to eradicate cancer cells.

The accuracy and efficiency of CAR T-cell therapy largely depend on the structure of the CARs. CARs consist of an extracellular antigen recognition domain, hinge region and transmembrane domain, which is riveted to an intracellular signaling domain. The extracellular domain is usually a single-chain variable fragment ( $\mathrm{scFv}$ ) derived from an antibody, with the ability to specifically target tumor cells with the tumor-specific antigen. The antibodies are usually derived from humans or mice or from humanization of murine antibodies. The hinge is the crystalline fragment $(\mathrm{Fc})$ region of immunoglobulin (Ig), mediating the antigen-antibody reaction and the elimination of antibodies. At the same time, it may provide a flexible connection between binding sites and contribute to the binding affinity. The transmembrane domain has the role of an anchor to the cell membrane and connects the ectodomain and endodomain, which affects the transmission of activating signals (10). The intracellular signaling domain, consisting of costimulatory domains and T-cell activation moieties, is involved in signal transduction, which is initiated by the binding of the extracellular domain to the antigen, in order to activate the immune system to eliminate the target cell (11).

CARs may be classified into four generations: The first generation is a fusion protein linked to the extracellular $\mathrm{ScFv}$ and $\mathrm{CD} 3 \zeta$ chain of the T-cell activation signal region, but cells lack long-term survival and robust proliferation. Therefore, by incorporating costimulatory endodomain molecules into $\mathrm{CD} 3 \xi$, such as $41 \mathrm{BB}$ and/or CD28, the persistence and antitumor ability of CAR T-cells in the body have been significantly improved (12). Of note, according to whether one or two co-stimulatory domains are added, the CARs are referred to as second- or third-generation CARs, respectively (Fig. 1) (13).

Until the emergence of the fourth generation of CAR T-cells redirected for universal cytokine-mediated killing, the exploration of the structure of CARs advanced a step further by adding an encoded vector on the basis of the previous structure to induce the expression of corresponding cytokines and enhance other effectors of the immune system in order to release cytokines in the immunosuppressive tumor microenvironment to recruit and activate more immune cells to strengthen the antitumor activity of CAR T-cells (14). Consequently, due to the high efficacy and selectivity, CAR T-cell therapy is becoming a promising novel therapeutic strategy which is inspiring increased research efforts. 


\section{Clinical applications of CAR T-cell therapy}

Hematological malignancies. CAR T-cell therapy holds great promise for hematological malignancies and is widely used in the management of lymphoma, leukemia and myeloma (15).

In August 2017, the first CAR T-cell therapy, Kymriah, was historically approved for the treatment of certain pediatric and young adult patients with B-cell acute lymphoblastic leukemia (B-ALL), with an overall response rate of $52 \%$ in a clinical trial (16). In October of the same year, a second CAR T-cell therapy named Yescarta was approved for the treatment of adult patients with certain types of relapsed/refractory $(\mathrm{R} / \mathrm{R})$ B-cell non-Hodgkin lymphoma. Both these therapies are antiCD19 CAR T-cell therapies with the ability to induce durable responses.

CD19 is a member of the Ig superfamily and is a type of transmembrane glycoprotein and a biomarker for lymphoma primarily expressed on malignant B-cells (17). As a popular immunotherapy target, CD19 is widely used in the treatment of hematological malignancies for its considerable efficacy. In a clinical trial using anti-CD19 CAR T-cell therapy for ALL, the response rate achieved was up to $81 \%$ with a complete remission rate of $78 \%$ (18).

Furthermore, CD-19-targeted CAR T-cell therapy may be applied for treating patients with chronic lymphocytic leukemia (CLL), but at present, it is mostly used in clinical trials and is lacking official approval (19). As a B-cell malignancy, CLL is the most common type of adult leukemia in western countries and is associated with defects of the immune system. There are several potentially effective treatment options available, but CLL cannot be cured with traditional therapies and the progression of the disease is inevitable. A previous study demonstrated that in patients with R/R CLL, CD19-directed CAR T-cells were able to clear tumor cells in the body with an overall response rate of $57 \%$ (20).

In addition to the CD19 antigen, novel targets are being continuously identified. In a clinical trial of CAR T-cell therapy targeting CD22, 73\% (11/15) of B-ALL patients achieved complete remission after receiving anti-CD22 CAR T-cell therapy (21). A retrospective analysis demonstrated that conventional CD19 may be combined with aberrant antigens, such as CD123, to form CARs with bi-specific antigens, which may significantly improve the efficacy when treating $R / R$ B-ALL (22). Furthermore, CD123 is considered a promising target for acute myeloid leukemia (AML) (23). It was previously demonstrated that CD33-targeted CAR T-cells may clear leukemia without causing myelosuppression in the treatment of AML (24).

In addition to leukemia, CAR T-cell therapy also demonstrated great potential in the management of multiple myeloma (MM). MM is a hematological malignancy with the secondhighest annual incidence among hematological tumors, caused by gene mutations during B-lymphocyte differentiation into plasma cells. In an experiment using biepitope-targeting CAR T-cells against $\mathrm{B}$-cell maturation antigen (BCMA) in 17 cases of R/R MM, the overall response rate reached $88.2 \%$ (25). As a transmembrane glycoprotein, BCMA belongs to the tumor necrosis factor receptor superfamily, which has an important role in long-term plasma cell survival and B-cell differentiation into plasma cells. In addition, BCMA is important for the survival of malignant plasma cells and its upregulation may promote MM-cell growth, while its downregulation inhibits MM-cell proliferation. Furthermore, BCMA is usually expressed at higher levels in malignant plasma cells compared to normal plasma cells, which may be an important factor for the effectiveness of CAR T-cell therapy when applied to patients with MM (26). Other clinical trials also reported promising results. A clinical study including patients with MM who received BCMA-targeting CAR T-cell therapy reported an overall response rate of $81 \%$ (27). In addition, CD38 and CD138 may be potential targets for MM treatment strategies $(28,29)$.

Solid tumors. CAR T-cell therapy has achieved great successes in treating hematological malignancies, but its application in solid tumors has not been as successful due to the lack of optimal antigens and the immunosuppressive tumor microenvironment (TME).

Continuous research and ongoing experiments aim at constructing solid tumor-associated antigens. For instance, CAR T-cells against human epidermal growth factor receptor 2 (HER2) may not only recognize and kill HER2 ${ }^{+}$breast cancer cells but also induce regression of experimental breast cancer in vivo (30). In addition, a previous study demonstrated that phosphatidylinositol 3 (GPC3)-targeted CAR T-cell therapy may also be a potential treatment for liver cancer (31). In a recent study, a CAR (hYP7) T-cell model was constructed, which was able to eliminate GPC3-positive hepatocellular cancer (HCC) cells, possibly by inducing perforin- and granzyme-mediated apoptosis or reducing Wnt signaling in tumor cells (32), emphasizing the potential of CAR T-cell therapy targeting GPC 3 in the treatment of patients with HCC. In addition, GPC3-targeting CAR T-cells expressing IL-12 have been constructed, which may significantly enhance the function of CAR T-cells with relatively fewer side effects (33).

A study demonstrated that prostate-specific membrane antigen and MUC1 may serve as potential targets in nonsmall cell lung cancer (34). Furthermore, mesothelin, anthrax toxin receptor 1 (ANTXR1; also known as tumor endothelial marker 8) and mucin $3 \mathrm{~A}$ are possible targets for CAR T-cell therapy in gastric adenocarcinomas (35). Anti-CD22/ anti-CD19 CAR T-cell therapy has been successfully used to treat R/R B-cell lymphoma involving the gastrointestinal tract. Out of 14 patients, 10 achieved an objective response and 7 patients achieved a complete response (36). Previous studies have indicated that programmed cell death protein 1 knockout may enhance the lytic activity of epidermal growth factor receptor variant III (EGFRvIII)-CAR T-cells against programmed death-ligand 1 EGFRvIII glioblastoma multiforme (GBM) cells, which may provide a novel therapeutic strategy for GBM (37). In addition, CAR T-cell therapy is also applied to treat solid tumor types, including pancreatic cancer, renal cell carcinoma, ovarian cancer, colorectal cancer and melanoma (38).

Regarding the use of CAR T-cells for the management of solid tumors, our group has also performed various preliminary investigations. A number of tumor antigens have been screened to be used for CAR T-cell therapy, among which, our previous study constructed a humanized protein tyrosine 


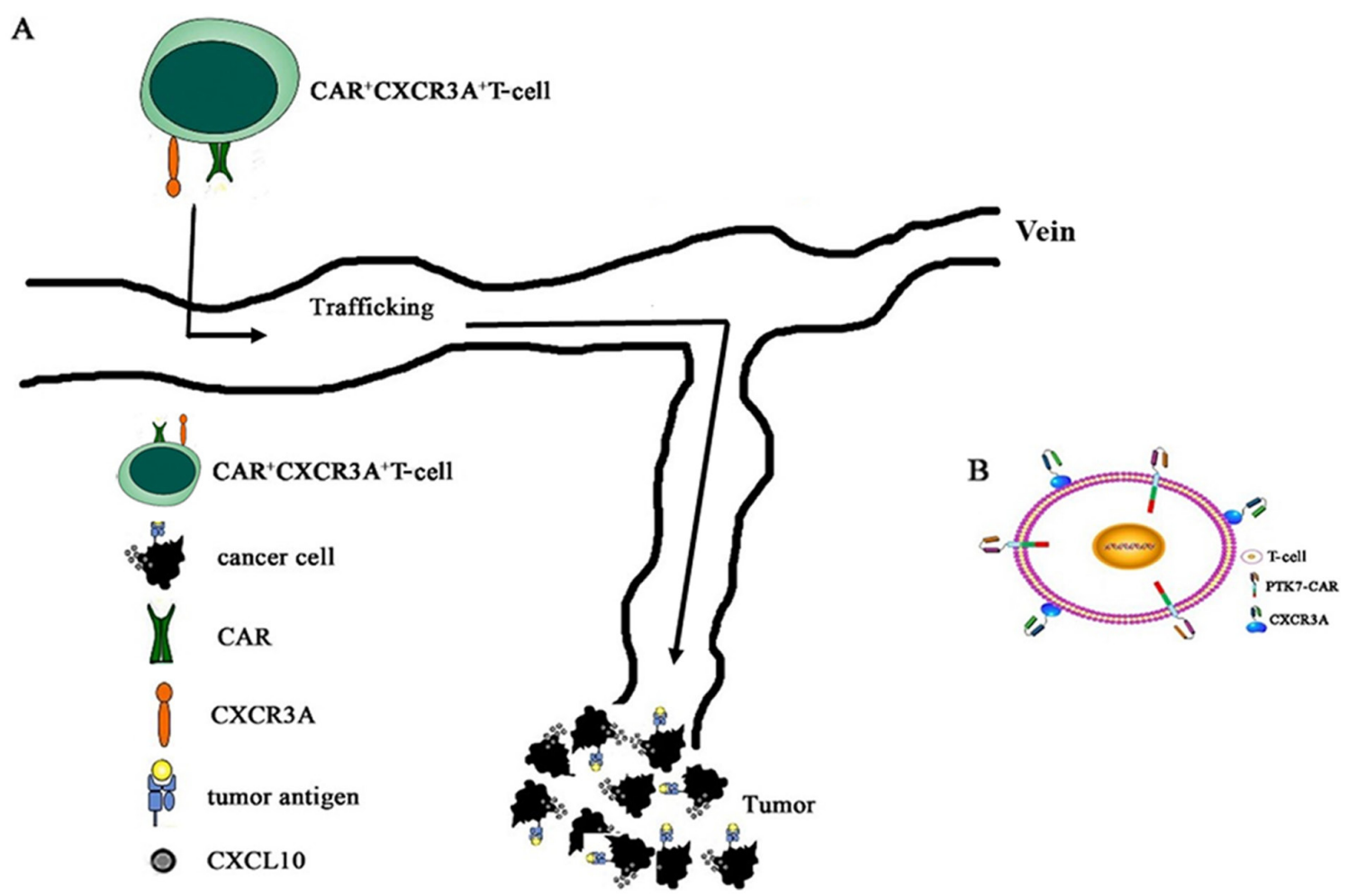

Figure 2. Schematic illustration of the mechanisms of action of CAR T-cells against esophageal squamous cell carcinoma. (A) Role of CAR T-cells in targeting PTK7 to treat esophageal squamous cell carcinoma. (B) CAR T-cells (CAR + CXCR3A + T-cells) expressing CXCR3A. CAR T-cells, chimeric antigen receptor-modified T-cells; PTK7, protein tyrosine kinase 7; CXCR/L, C-X-C motif chemokine receptor/ligand.

kinase 7-CAR through genetic engineering to perform genetic modification of lymphocytes (Fig. 2) (39).

\section{Current challenges and treatment principles of CAR T-cell therapy}

Cytokine release syndrome (CRS). CRS is a systemic inflammatory state that occurs as a result of robust immune activation. As one of the most frequent serious adverse events associated with CAR T-cell therapy, CRS is reported in patients who receive CAR T-cell therapy with an incidence of 57-93\%, depending on the type of immunotherapy and the disease burden (40).

CRS is mainly triggered by a large number of cytokines and inflammatory factors, and interleukin (IL)-6 has a key role in mediating CRS. However, the levels of other cytokines also increase over the course of CRS, including IL-2, interferon- $\gamma$, tumor necrosis factor, GM-CSF, IL-5, IL-8 and IL-10 (41).

As a systemic inflammatory response, CRS involves multiple systems and has various aspects, such as circulatory, breathing, urinary and digestive system disorders. The patient may develop fever, skin rash, cardiac dysfunction, respiratory failure, renal failure, nausea, vomiting, hepatic dysfunction, disseminated intravascular coagulopathy and neurotoxicity after undergoing CAR T-cell therapy $(42,43)$.

Although most cases of CRS are self-limited, it may prove life-threatening without timely and effective treatment (44). For instance, respiratory symptoms are common in mild cases. CRS may manifest as cough and tachypnea, but it may progress to acute respiratory distress syndrome with hypoxemia and dyspnea. When respiratory dysfunction occurs, mechanical ventilation is usually required. Similarly, if cardiovascular (CV) side effects occur, patients must be promptly transferred to the intensive care unit where fluids and pressors may be administered to effectively manage tachycardia and hypotension (45). A study on CRS and cardiotoxicity caused by CAR T-cell therapy reported that heart injury and CV events are common in adult patients following CAR T-cell therapy, but a shorter time from CRS onset to the utilization of tocilizumab was associated with a lower rate of CV events (46). Therefore, it is crucial to ensure timely detection and treatment for patients receiving CAR T-cell to prevent further damage to the body from CRS.

Of note, patients with mild and moderate symptoms may require supportive measures, such as antibiotics and antipyretics, while tocilizumab is usually required in severe cases. As the first-line medicine in the clinical treatment of CRS, tocilizumab may ameliorate CRS by antagonising IL-6 (47). Furthermore, tocilizumab has already demonstrated clinical efficacy in treating CRS, without compromising the antitumor effects, and clinical improvement is usually observed within hours. As a consequence, tocilizumab was approved by the FDA in August 2017 and is currently considered as the mainstay of treatment for severe CRS (48).

Of note, both tocilizumab and steroids may be used to treat CRS. However, the use of tocilizumab is favored over corticosteroids, as the latter may adversely affect the treatment efficacy (49).

Regarding the treatment of $\mathrm{R} / \mathrm{R}$ chronic leukemia, studies have confirmed that the combination of ibrutinib and anti-CD19 CAR T-cell immunotherapy was able to effectively improve the antitumor efficacy of CAR T-cells and reduce the incidence 
of CRS (50). The use of dual-target chimeric antigen receptor T-cells may also be considered. A case report on a patient with R/R primary central nervous system (PCNS) diffuse large B-cell lymphoma treated with anti-CD19- and antiCD70-specific CAR T-cell therapy indicated that CAR T-cells were able to effectively target PCNS lymphomas and maintain disease-free survival without inducing CRS (51). Another study reported that, with the use of real-time monitoring of cytokines, CAR T-cell therapy against CD19 may be safely applied in patients with $\mathrm{R} / \mathrm{R}$ leukemia (52).

Immune effector-related neurotoxicity syndrome (ICANS). ICANS is the second most common complication of CAR T-cell therapy with an incidence of $12-55 \%$.

ICANS may manifest with a diverse range of symptoms, spanning from mild confusion with tremor, dysgraphia, mild expressive aphasia and apraxia, to seizures, somnolence, global aphasia and, in a minority of cases, death $(53,54)$. Fatal cerebral edema has also been reported (55). Another previous study indicated that patients with a higher tumor burden were more likely to develop neurotoxic symptoms when receiving high-dose CAR T-cell therapy. Of the 25 adult patients who developed ICANS following CAR T-cell therapy, 12 (48\%) developed grade 1-2 neurotoxicity, whereas 13 (52\%) presented with grade 3-4 neurotoxicity (56). In a phase I study including 53 adult patients with B-ALL treated with CD19 CAR T-cell therapy, grade 1 and 2 neurotoxicity was observed in 11 patients, and grade 3 and 4 neurotoxicity was observed in 22 patients (57). Although the pathophysiology of the neurotoxicity has yet to be fully elucidated, there is evidence of breakdown of the blood-brain barrier in patients with severe neurotoxicity and the protein concentration in the cerebrospinal fluid (CSF) is correlated with the grade of neurotoxicity, indicating that there is increased permeability of the bloodCSF barrier during ICANS. It was previously reported that the neurotoxicity of CAR T-cell therapy may be associated with the proliferation of CAR T-cells in the central nervous system. A phase I study enrolled 21 pediatric and young adult patients (aged 1-30 years) with R/R ALL or non-Hodgkin lymphoma (58) and reported that those who developed neurotoxicity had higher concentrations of CAR T-cells in their CSF. In this trial, the numbers of CAR T-cells in the CSF of patients exhibiting neurotoxicity were higher compared with those in patients who did not receive this therapy. Similar to CRS, ICANS is also self-limited and the therapeutic interventions are mainly supportive, aimed at attenuating the immune system response by using steroids (59).

Tumor lysis syndrome (TLS). TLS refers to the metabolic disorder caused by the excessive release of uric acid, potassium and phosphorus following the death of a large number of cancer cells within a short time period.

In general, the symptoms are non-specific, including nausea, fatigue, numbness, hallucinations, cramps and palpitations. TLS is a common tumor emergency and, if left untreated, may lead to kidney failure, heart failure and death in severe cases (60). When patients with a high tumor burden receive CAR T-cell therapy, plenty of tumor cells are killed by CAR T-cells within a short period, releasing their contents into the blood and leading to the development of TLS. Therefore, when administering CAR T-cell therapy, the results of blood tests and the characteristics of the cancer should be taken into consideration to evaluate the risk of developing TLS. Furthermore, adequate preventive measures are required to reduce the risk of TLS $(61,62)$.

Homing disorders. Another cause for the lack of response in numerous patients receiving CAR T-cell therapy is the poor homing of T-cells into tumors.

This may be caused by the lack of optimal tumor-associated or tumor-specific antigens. An optimal target should have wide tumor coverage and be highly specific for the tumor in order to ensure safety and efficacy. Unlike hematological cancer cells carrying homogeneous target antigens, the antigens on solid tumor cells are heterogeneous and may differ between the primary and metastatic stages of the same tumor; therefore, finding a suitable antigen may prove difficult (63).

The second reason for homing disorders is the immunosuppressive TME. The lack of sufficient blood supply, low $\mathrm{pH}$ and certain cytokines prevent the continued antitumor activity of the immune system (64). In addition, fibroblasts and myeloid cells may form a physical barrier around the tumor to prevent CAR T-cells from entering the TME. Furthermore, a variety of chemokines secreted by solid tumors may lead to immune escape and make the penetration of CAR T-cells less efficient. As a consequence, it is difficult for CAR T-cells to adequately home to and infiltrate the tumor.

In order to solve these problems, methods for improving the treatment efficacy of CAR T-cells in solid tumors have been under investigation. In a study by Katz et al (65), CAR T-cell therapy was applied to patients with liver metastases through percutaneous hepatic artery infusions, which limited extrahepatic toxicity while optimizing treatment efficacy. Other methods, such as engineering CAR T-cells to express cytokines, such as IL-7 and CCL19, may enhance the efficacy of this therapeutic application by recruiting T-cells and DCs to tumor tissues (66). In addition, as a chemokine receptor, $\mathrm{C}-\mathrm{X}-\mathrm{C}$ motif chemokine receptor 3 has a key role in activating $\mathrm{T}$-cells and is involved in the development and persistence of tissue-resident memory CD8 ${ }^{+}$T-cells (67).

Poor proliferation and short-term persistence. As well as the antigen loss, poor persistence of the infused CAR T-cells is one of the major causes of treatment failure, resulting from programmed cell death or exhaustion of T-cells. Of note, the exhaustion of T-cells is complex and may be affected by a variety of factors, including the starting state of T-cells, patient age and the TME (68). It has been reported that CAR T-cells may be functionally suppressed under adverse conditions, including poor blood supply, low pH and hypoxia (69). Furthermore, tumors secrete cytokines to recruit various types of immunosuppressive cells that may secrete antiinflammatory cytokines and/or express ligands for immune checkpoint receptors, which potentially causes the exhaustion of CAR T-cells (70). As a consequence, these negative regulators lead to the poor expansion or short-term persistence of CAR T-cells.

On-target, off-tumor toxicity. On-target off-tumor toxicity is an adverse effect associated with CAR T-cell-induced injury 
of normal tissues. Since tumor-associated antigens may also be weakly expressed in normal tissues (71), potential damage of normal tissues may occur during immunotherapy (72). A severe event was reported during the treatment of a patient with colon cancer metastasis to the lungs and liver (73). In that case, the patient developed respiratory distress within $15 \mathrm{~min}$ after the CAR T-cell infusion and succumbed to the condition 5 days later. The final conclusion was that the patient's death was caused by the localization of a large number of CAR T-cells in the lungs and severe injury of the pulmonary epithelial cells.

Current research indicates that the introduction of suicide gene systems may be used to address the possible on-target offtumor toxicity (74). Furthermore, by engineering CAR T-cells to express two or more receptors, such CARs may recognize the tumor cells more specifically, while normal tissues are protected as they only express one antigen (75).

\section{Conclusions and outlook}

Compared with traditional therapy, immunotherapy exhibits higher specificity and stronger antitumor efficacy. Furthermore, this field is rapidly advancing due to continuous scientific breakthroughs.

Although CAR T-cell therapy is rapidly developing and appears to hold great promise, several issues remain to be resolved, such as increasing the ability of CAR T-cells to infiltrate solid tumor tissues, enhancing their specificity when applied to various solid tumors and increasing their ability to cope with the immunosuppressive TME.

With the continuous development of tumor immunotherapy, the related anticancer drugs are expected to be more widely used in the future. Therefore, the incidence of immunerelated adverse reactions is expected to increase over the next few years and effectively reducing various adverse reactions should be the focus of future research. In addition, traditional CARs have a fixed design, which limits their clinical application due to the high manufacturing cost, lengthy production process and an inherent risk of production failure; therefore, the design of a universal CAR represents a new goal as well as a significant challenge (76).

\section{Acknowledgements}

Not applicable.

\section{Funding}

This work was supported by the National College Student Innovation and Entrepreneurship Training Program (grant no. 202010366031), the Science and Technology Project of the Health Planning Committee of Sichuan (grant nos. 19PJ057 and 20PJ177), Sichuan Science and Technology Program (grant nos. 2018SZ0377 and 2018JY0219) and Nanchong Science and Technology Program (grant nos. 19SXHZ0451 and 18SXHZ0366).

\section{Availability of data and materials}

Not applicable.

\section{Authors' contributions}

KL and FL designed the review. YL and GS performed the literature search, selection of articles and wrote the paper. SL checked every edition and made revisions. All authors read and approved the final manuscript.

\section{Ethics approval and consent to participate}

Not applicable.

\section{Patient consent for publication}

Not applicable.

\section{Competing interests}

The authors declare that they have no competing interests.

\section{References}

1. Panagopoulou TI and Rafiq QA: CAR-T immunotherapies: Biotechnological strategies to improve safety, efficacy and clinical outcome through CAR engineering. Biotechnol Adv 37: 107411, 2019

2. Couzin-Frankel J: Breakthrough of the year 2013. Cancer immunotherapy. Science 342: 1432-1433, 2013.

3. Tan S, Li D and Zhu X: Cancer immunotherapy: Pros, cons and beyond. Biomed Pharmacother 124: 109821, 2020.

4. Peng M, Mo Y, Wang Y, Wu P, Zhang Y, Xiong F, Guo C, Wu X, $\mathrm{Li} Y, \mathrm{Li} X$, et al: Neoantigen vaccine: An emerging tumor immunotherapy. Mol Cancer 18: 128, 2019.

5. Yáñez L, Sánchez-Escamilla M and Perales MA: CAR T cell toxicity: Current management and future directions. HemaSphere 3: e186, 2019.

6. Ma CC, Wang ZL, Xu T, He ZY and Wei YQ: The approved gene therapy drugs worldwide: from 1998 to 2019. Biotechnol Adv 40: $107502,2020$.

7. Mullard A: FDA approves first CAR T therapy. Nat Rev Drug Discov 16: 669, 2017.

8. No authors listed: FDA approves second CAR T-cell therapy. Cancer Discov 8: 5-6, 2018.

9. Huang $X$ and Yang Y: Driving an improved CAR for cancer immunotherapy. J Clin Invest 126: 2795-2798, 2016.

10. Huang R, Li X, He Y, Zhu W, Gao L, Liu Y, Gao L, Wen Q, Zhong JF, Zhang C, et al: Recent advances in CAR-T cell engineering. J Hematol Oncol 13: 86, 2020.

11. Jackson HJ, Rafiq S and Brentjens RJ: Driving CAR T-cells forward. Nat Rev Clin Oncol 13: 370-383, 2016.

12. Depil S, Duchateau P, Grupp SA, Mufti G and Poirot L: 'Off-the-shelf' allogeneic CAR T cells: Development and challenges. Nat Rev Drug Discov 19: 185-199, 2020.

13. Schmidts A and Maus MV: Making CAR T cells a solid option for solid tumors. Front Immunol 9: 2593, 2018.

14. Chmielewski M and Abken H: TRUCKs: The fourth generation of CARs. Expert Opin Biol Ther 15: 1145-1154, 2015.

15. Li D, Li X, Zhou WL, Huang Y, Liang X, Jiang L, Yang X, Sun J, Li Z, Han WD, et al: Genetically engineered T cells for cancer immunotherapy. Signal Transduct Target Ther 4: 35, 2019.

16. Schuster SJ, Bishop MR, Tam CS, Waller EK, Borchmann P, McGuirk JP, Jäger U, Jaglowski S, Andreadis C, Westin JR, et al; JULIET Investigators: Tisagenlecleucel in adult relapsed or refractory diffuse large B-cell lymphoma. N Engl J Med 380: 45-56, 2019.

17. Yamada S, Kaneko MK, Sayama Y, Asano T, Sano M, Yanaka M, Nakamura T, Okamoto S, Handa S, Komatsu Y, et al: Development of novel mouse monoclonal antibodies against human CD19. Monoclon Antib Immunodiagn Immunother 39: 45-50, 2020.

18. Schubert ML, Hückelhoven A, Hoffmann JM, Schmitt A, Wuchter P, Sellner L, Hofmann S, Ho AD, Dreger P and Schmitt M: Chimeric antigen receptor T cell therapy targeting CD19-positive leukemia and lymphoma in the context of stem cell transplantation. Hum Gene Ther 27: 758-771, 2016. 
19. Iovino L and Shadman M: Novel therapies in chronic lymphocytic leukemia: A rapidly changing landscape. Curr Treat Options Oncol 21: 24, 2020.

20. Porter DL, Hwang WT, Frey NV, Lacey SF, Shaw PA, Loren AW, Bagg A, Marcucci KT, Shen A, Gonzalez V, et al: Chimeric antigen receptor $\mathrm{T}$ cells persist and induce sustained remissions in relapsed refractory chronic lymphocytic leukemia. Sci Transl Med 7: 303ra139, 2015.

21. Fry TJ, Shah NN, Orentas RJ, Stetler-Stevenson M, Yuan CM, Ramakrishna S, Wolters P, Martin S, Delbrook C, Yates B, et al: CD22-targeted CAR T cells induce remission in B-ALL that is naive or resistant to CD19-targeted CAR immunotherapy. Nat Med 24: 20-28, 2018

22. Liu J, Tan X, Ma YY, Liu Y, Gao L, Gao L, Kong P, Peng XG, Zhang $\mathrm{X}$ and Zhang C: Study on the prognostic value of aberrant antigen in patients with acute B lymphocytic leukemia. Clin Lymphoma Myeloma Leuk 19: e349-e358, 2019.

23. Qin H, Edwards JP, Zaritskaya L, Gupta A, Mu CJ, Fry TJ, Hilbert DM and LaFleur DW: Chimeric antigen receptors incorporating D domains targeting CD123 direct potent mono- and bi-specific antitumor activity of T cells. Mol Ther 27: 1262-1274, 2019.

24. Borot F, Wang H, Ma Y, Jafarov T, Raza A, Ali AM and Mukherjee S: Gene-edited stem cells enable CD33-directed immune therapy for myeloid malignancies. Proc Natl Acad Sci USA 116: 11978-11987, 2019.

25. Xu J, Chen LJ, Yang SS, Sun Y, Wu W, Liu YF, Xu J, Zhuang Y, Zhang W, Weng XQ, et al: Exploratory trial of a biepitopic CAR T-targeting B cell maturation antigen in relapsed/refractory multiple myeloma. Proc Natl Acad Sci USA 116: 9543-9551, 2019.

26. D'Agostino M and Raje N: Anti-BCMA CAR T-cell therapy in multiple myeloma: Can we do better? Leukemia 34: 21-34, 2020.

27. Brudno JN, Maric I, Hartman SD, Rose JJ, Wang M, Lam N, Stetler-Stevenson M, Salem D, Yuan C, Pavletic S, et al: T cells genetically modified to express an anti-B-cell maturation antigen chimeric antigen receptor cause remissions of poor-prognosis relapsed multiple myeloma. J Clin Oncol 36: 2267-2280, 2018.

28. Morandi F, Horenstein AL, Costa F, Giuliani N, Pistoia V and Malavasi F: CD38: A target for immunotherapeutic approaches in multiple myeloma. Front Immunol 9: 2722, 2018.

29. Vasuthasawat A, Yoo EM, Trinh KR, Lichtenstein A, Timmerman JM and Morrison SL: Targeted immunotherapy using anti-CD138-interferon $\alpha$ fusion proteins and bortezomib results in synergistic protection against multiple myeloma. MAbs 8: 1386-1397, 2016

30. Sun M, Shi H, Liu C, Liu J, Liu X and Sun Y: Construction and evaluation of a novel humanized HER2-specific chimeric receptor. Breast Cancer Res 16: R61, 2014.

31. Gao H, Li K, Tu H, Pan X, Jiang H, Shi B, Kong J, Wang H, Yang S, Gu J, et al: Development of T cells redirected to glypican-3 for the treatment of hepatocellular carcinoma. Clin Cancer Res 20: 6418-6428, 2014

32. Li D, Li N, Zhang YF, Fu H, Feng M, Schneider D, Su L, Wu X, Zhou J, Mackay S, et al: Persistent polyfunctional chimeric antigen receptor $\mathrm{T}$ cells that target glypican 3 eliminate orthotopic hepatocellular carcinomas in mice. Gastroenterology 158: 2250-2265.e20, 2020.

33. Liu Y, Di S, Shi B, Zhang H, Wang Y, Wu X, Luo H, Wang H, $\mathrm{Li} \mathrm{Z}$ and Jiang H: Armored inducible expression of IL-12 enhances antitumor activity of glypican-3-targeted chimeric antigen receptor-engineered T cells in hepatocellular carcinoma. J Immunol 203: 198-207, 2019.

34. Wei X, Lai Y, Li J, Qin L, Xu Y, Zhao R, Li B, Lin S, Wang S, Wu Q, et al: PSCA and MUC1 in non-small-cell lung cancer as targets of chimeric antigen receptor T cells. OncoImmunology 6: e1284722, 2017.

35. Sotoudeh M, Shirvani SI, Merat S, Ahmadbeigi N and Naderi M: MSLN (Mesothelin), ANTXR1 (TEM8), and MUC3A are the potent antigenic targets for CAR T cell therapy of gastric adenocarcinoma. J Cell Biochem 120: 5010-5017, 2019.

36. Zeng C, Cheng J, Li T, Huang J, Li C, Jiang L, Wang J, Chen L, Mao X, Zhu L, et al: Efficacy and toxicity for CD22/ CD19 chimeric antigen receptor T-cell therapy in patients with relapsed/refractory aggressive B-cell lymphoma involving the gastrointestinal tract. Cytotherapy 22: 166-171, 2020.

37. Zhu H, You Y, Shen Z and Shi L: EGFRvIII-CAR-T cells with PD-1 knockout have improved anti-glioma activity. Pathol Oncol Res 26: 2135-2141, 2020.
38. Zhang BL, Li D, Gong YL, Huang Y, Qin DY, Jiang L, Liang X, Yang X, Gou HF, Wang YS, et al: Preclinical evaluation of chimeric antigen receptor-modified $\mathrm{T}$ cells specific to epithelial cell adhesion molecule for treating colorectal cancer. Hum Gene Ther 30: 402-412, 2019.

39. Liu K, Song G, Zhang X, Li Q, Zhao Y, Zhou Y, Xiong R, Hu X, Tang Z and Feng G: PTK7 is a novel oncogenic target for esophageal squamous cell carcinoma. World J Surg Oncol 15: 105, 2017.

40. Gutgarts V, Jain T, Zheng J, Maloy MA, Ruiz JD, Pennisi M, Jaimes EA, Perales MA and Sathick J: Acute kidney injury after CAR-T cell therapy: Low incidence and rapid recovery. Biol Blood Marrow Transplant 26: 1071-1076, 2020.

41. Aldoss I, Khaled SK, Budde E and Stein AS: Cytokine release syndrome with the novel treatments of acute lymphoblastic leukemia: Pathophysiology, prevention, and treatment. Curr Oncol Rep 21: 4, 2019.

42. Riegler LL, Jones GP and Lee DW: Current approaches in the grading and management of cytokine release syndrome after chimeric antigen receptor T-cell therapy. Ther Clin Risk Manag 15: 323-335, 2019.

43. Shimabukuro-Vornhagen A, Gödel P, Subklewe M, Stemmler HJ, Schlößer HA, Schlaak M, Kochanek M, Böll B and von BergweltBaildon MS: Cytokine release syndrome. J Immunother Cancer 6: 56,2018

44. Chou CK and Turtle CJ: Assessment and management of cytokine release syndrome and neurotoxicity following CD19 CAR-T cell therapy. Expert Opin Biol Ther 20: 653-664, 2020.

45. Thakar MS, Kearl TJ and Malarkannan S: Controlling cytokine release syndrome to harness the full potential of CAR-based cellular therapy. Front Oncol 9: 1529, 2020.

46. Alvi RM, Frigault MJ, Fradley MG, Jain MD, Mahmood SS, Awadalla M, Lee DH, Zlotoff DA, Zhang L, Drobni ZD, et al: Cardiovascular events among adults treated with chimeric antigen receptor T-cells (CAR-T). J Am Coll Cardiol 74: 3099-3108, 2019

47. Liu D and Zhao J: Cytokine release syndrome: Grading, modeling, and new therapy. J Hematol Oncol 11: 121, 2018.

48. Gödel P, Shimabukuro-Vornhagen A and von BergweltBaildon M: Understanding cytokine release syndrome. Intensive Care Med 44: 371-373, 2018.

49. Acharya UH, Dhawale T, Yun S, Jacobson CA, Chavez JC, Ramos JD, Appelbaum J and Maloney DG: Management of cytokine release syndrome and neurotoxicity in chimeric antigen receptor (CAR) T cell therapy. Expert Rev Hematol 12: 195-205, 2019.

50. Gauthier J, Hirayama AV, Purushe J, Hay KA, Lymp J, Li DH, Yeung CCS, Sheih A, Pender BS, Hawkins RM, et al: Feasibility and efficacy of CD19-targeted CAR T cells with concurrent ibrutinib for CLL after ibrutinib failure. Blood 135: 1650-1660, 2020.

51. Tu S, Zhou X, Guo Z, Huang R, Yue C, He Y, Li M, Chen Y, Liu Y, Chang LJ, et al: CD19 and CD70 dual-target chimeric antigen receptor T-cell therapy for the treatment of relapsed and refractory primary central nervous system diffuse large B-cell lymphoma. Front Oncol 9: 1350, 2019.

52. Shen D, Song H, Xu X, Xu W, Wang D, Liang J, Fang M, Liao C, Chen X, Li S, et al: Chimeric antigen receptor T cell therapy can be administered safely under the real-time monitoring of Th1/ Th2 cytokine pattern using the cytometric bead array technology for relapsed and refractory acute lymphoblastic leukemia in children. Pediatr Hematol Oncol 37: 288-299, 2020.

53. Cornillon J, Hadhoum N, Roth-Guepin G, Quessar A, Platon L, Ouachée-Chardin M, Nicolas-Virelizier E, Naudin J, Moreau AS, Masouridi-Levrat S, et al: Management of CAR-T cell-related encephalopathy syndrome in adult and pediatric patients: Recommendations of the French Society of Bone Marrow transplantation and cellular Therapy (SFGM-TC). Bull Cancer 107: S12-S17, 2020 (In French).

54. Kennedy LB and Salama AKS: A review of cancer immunotherapy toxicity. CA Cancer J Clin 70: 86-104, 2020

55. Turtle CJ, Hay KA, Hanafi LA, Li D, Cherian S, Chen X, Wood B, Lozanski A, Byrd JC, Heimfeld S, et al: Durable molecular remissions in chronic lymphocytic leukemia treated with CD19specific chimeric antigen receptor-modified $\mathrm{T}$ cells after failure of Ibrutinib. J Clin Oncol 35: 3010-3020, 2017.

56. Karschnia P, Jordan JT, Forst DA, Arrillaga-Romany IC, Batchelor TT, Baehring JM, Clement NF, Gonzalez Castro LN, Herlopian A, Maus MV, et al: Clinical presentation, management, and biomarkers of neurotoxicity after adoptive immunotherapy with CAR T cells. Blood 133: 2212-2221, 2019. 
57. Santomasso BD, Park JH, Salloum D, Riviere I, Flynn J, Mead E, Halton E, Wang X, Senechal B, Purdon T, et al: Clinical and biological correlates of neurotoxicity associated with CAR T-cell therapy in patients with B-cell acute lymphoblastic leukemia. Cancer Discov 8: 958-971, 2018.

58. Lee DW, Kochenderfer JN, Stetler-Stevenson M, Cui YK, Delbrook C, Feldman SA, Fry TJ, Orentas R, Sabatino M, Shah NN, et al: T cells expressing CD19 chimeric antigen receptors for acute lymphoblastic leukaemia in children and young adults: A phase 1 dose-escalation trial. Lancet 385 $517-528,2015$

59. Oved JH, Barrett DM and Teachey DT: Cellular therapy: Immune-related complications. Immunol Rev 290: 114-126, 2019.

60. Gupta A and Moore JA: Tumor lysis syndrome. JAMA Oncol 4: 895, 2018.

61. Feldmann A, Arndt C, Koristka S, Berndt N, Bergmann R and Bachmann MP: Conventional CARs versus modular CARs. Cancer Immunol Immunother 68: 1713-1719, 2019.

62. Neelapu SS: Managing the toxicities of CAR T-cell therapy. Hematol Oncol 37 (Suppl 1): 48-52, 2019.

63. Xia AL, Wang XC, Lu YJ, Lu XJ and Sun B: Chimeric-antigen receptor T (CAR-T) cell therapy for solid tumors: Challenges and opportunities. Oncotarget 8: 90521-90531, 2017.

64. Greenbaum U, Yalniz FF, Srour SA, Rezvani K, Singh H, Olson A, Blumenschein G Jr, Hong DS, Shpall EJ and Kebriaei P: Chimeric antigen receptor therapy: How are we driving in solid tumors? Biol Blood Marrow Transplant 26: 1759-1769, 2020.

65. Katz SC, Burga RA, McCormack E, Wang LJ, Mooring W, Point GR, Khare PD, Thorn M, Ma Q, Stainken BF, et al: Phase I hepatic immunotherapy for metastases study of intra-arterial chimeric antigen receptor-modified T-cell therapy for $\mathrm{CEA}^{+}$liver metastases. Clin Cancer Res 21: 3149-3159, 2015.

66. Adachi K, Kano Y, Nagai T, Okuyama N, Sakoda Y and Tamada K: IL-7 and CCL19 expression in CAR-T cells improves immune cell infiltration and CAR-T cell survival in the tumor. Nat Biotechnol 36: 346-351, 2018.

67. Alanio C, Barreira da Silva R, Michonneau D, Bousso P, Ingersoll MA and Albert ML: CXCR3/CXCL10 Axis Shapes Tissue Distribution of Memory Phenotype CD ${ }^{+} \mathrm{T}$ Cells in Nonimmunized Mice. J Immunol 200: 139-146, 2018.
68. Liu L, Bi E, Ma X, Xiong W, Qian J, Ye L, Su P, Wang Q, Xiao L, Yang M, et al: Enhanced CAR-T activity against established tumors by polarizing human T cells to secrete interleukin-9. Nat Commun 11: 5902, 2020.

69. Knochelmann HM, Smith AS, Dwyer CJ, Wyatt MM, Mehrotra S and Paulos CM: CAR T Cells in solid tumors: Blueprints for building effective therapies. Front Immunol 9: 1740, 2018.

70. Morgan MA and Schambach A: Engineering CAR-T cells for improved function against solid tumors. Front Immunol 9: 2493, 2018.

71. Yu S, Yi M, Qin S and Wu K: Next generation chimeric antigen receptor T cells: Safety strategies to overcome toxicity. Mol Cancer 18: 125, 2019.

72. Akbari P, Huijbers EJM, Themeli M, Griffioen AW and van Beijnum JR: The tumor vasculature an attractive CAR T cell target in solid tumors. Angiogenesis 22: 473-475, 2019.

73. Morgan RA, Yang JC, Kitano M, Dudley ME, Laurencot CM and Rosenberg SA: Case report of a serious adverse event following the administration of $\mathrm{T}$ cells transduced with a chimeric antigen receptor recognizing ERBB2. Mol Ther 18: 843-851, 2010.

74. Gargett $T$ and Brown MP: The inducible caspase- 9 suicide gene system as a 'safety switch' to limit on-target, off-tumor toxicities of chimeric antigen receptor T cells. Front Pharmacol 5: 235, 2014.

75. Kloss CC, Condomines M, Cartellieri M, Bachmann M and Sadelain M: Combinatorial antigen recognition with balanced signaling promotes selective tumor eradication by engineered T cells. Nat Biotechnol 31: 71-75, 2013.

76. Liu D, Zhao J and Song Y: Engineering switchable and programmable universal CARs for CAR T therapy. J Hematol Oncol 12: 69, 2019.

This work is licensed under a Creative Commons Attribution-NonCommercial-NoDerivatives 4.0 International (CC BY-NC-ND 4.0) License. 